\title{
Dispatch coordination between high-speed and conventional rail systems
}

\author{
Qiyuan PENG ${ }^{*}$, Chao WEN \\ School of Transportation and Logistics, Southwest Jiaotong University, Chengdu 610031, China
}

\begin{abstract}
Cross-line trains, as a link between high-speed and conventional rail networks, will increase the complexity of transport organization and lead to significant challenges in dispatch coordination between the two systems. Based on the characteristics of high-speed transport organization, this paper deals with the necessity of dispatch coordination between high-speed and conventional lines from the following two perspectives: the operation of cross-line trains and work coordination in connection stations. An adjustment model for the operation of high-speed trains, taking cross-line trains into account, is established. Finally, the dispatch system is described in terms of construction and process. Methods for organizing dispatch are proposed, and the processes of coordination adjustment under normal and unexpected situations are analyzed. The discussion in this paper may serve as a theoretical basis for the development of high-speed rail dispatch systems.
\end{abstract}

Key words: high-speed rail; conventional rail; dispatch; coordination

(C) 2011 JMT. All rights reserved.

\section{Introduction}

$\mathrm{S}$ ince high-speed (HS) and conventional rails are two independent, but inter-related systems, the combination of high-speed trains and cross-line trains is considered a proper transport mode for a completely accessible railway network. With this mode, one may not only expand the accessibility of high-speed passenger transport, but may also make full use of the capacity of high-speed and conventional rail networks. However, cross-line trains, as the link between high-speed and conventional rail networks, will increase the complexity of transport organization, and present a significant challenge to dispatch coordination between the two systems. Work organization in connection stations is directly related to the degree of connection between high-speed and conventional rail networks, and is one of the main problems of transport dispatch. Theories of dispatch coordination between high-speed and conventional rail networks is of practical significance for optimizing transport organization, improving dispatch efficiency, and ensuring traffic safety. In 1973, Szpigel [1] first proposed an optimal train schedule, using linear programming and the branch-and-bound method for train sched-

Received Jan. 19, 2011; revision accepted Mar. 8, 2011

*Corresponding author. Tel.: +86-28-87600750

Email: qiyuan-peng@swjtu.edu.cn (Q.Y. PENG)

doi: 10.3969/j.issn.2095-087X.2011.01.004 uling on a single line. In 1983, Araya [2] put forward an optimal rescheduling for online train control in perturbation. Then Jovanovic [3] proposed the introduction of a heuristic technology into integer programming for train dispatch. This method significantly reduced the number of search nodes, and thus greatly improved the calculation speed. In China, studies of the optimization of railway dispatch have focused on establishing real-time models for the adjustment of train operation and for designing the related algorithms, with the optimization objectives as follows: highest rate of punctuality [4], least delay time [5], higher average travel speed [6], and improvement of the satisfaction of the passengers during the train operation [7], etc. Nie [8] investigated train operation adjustment in high-speed lines, and proposed the general principles for high-speed rail dispatch. Xie [9] studied key issues of train control in high-speed lines, such as the train operation adjustment model, the optimal adjustment of electric multiple units (EMUs), and optimization research for utilization of arrival and departure tracks. Xie established the framework of highspeed rail dispatch system.

However, conventional studies have focused on highspeed and conventional rail systems independently, without full consideration of the dispatch coordination between the two systems. In this paper, the coordination demands of high-speed and conventional rails, the optimization model of dispatch coordination, and the establishment of organization are studied. 


\section{Bor: Characteristics of high-speed rail trans- port organization}

Because of its superiority in technology and economy, the high-speed rail system has some special features regarding transport organization that are different from the conventional rail system:

(1) In China, both the high-speed trains and the crossline trains, trains with different speeds, run on the same high-speed line. Cross-line transport takes into account the characteristics of passenger flow, which can effectively ease the limited capacity of the conventional rail system and bring the capacity of high-speed rail system into full play, despite the unsaturated flow in the beginning.

(2) For adjusting train operation on the conventional line, freight trains usually need to wait or avoid passenger trains unconditionally. In high-speed rail systems, however, in order to ensure the travel speed and punctuality rate of each train, the numbers of overtaking and refuges should be equal for trains at the same level, and a passenger train should not wait in a train station for a long time.

(3) In order to ensure safety during the operation of high-speed rail systems, a long-time comprehensive maintenance window (more than 2 hours for ballastless track, 4 hours for ballasted track) needs to remain open [10], and the affected zone around the window has a significant impact on the line capacity as well. Generally, high-speed passenger trains are not allowed to wait for long during the maintenance window period. The operation of cross-line and evening-morning passenger trains is one of the difficult problems in the organization of high-speed rail networks.

(4) Compared to the conventional rail system, the high- speed rail system has the prominent characteristic of peak and non-peak periods of passenger flow, which requires more rigorous operation conditions. Meanwhile, as high-speed rail cannot be interconnected with some conventional rails, a large number of passengers need to transfer in connection stations. Therefore, the problems of ensuring rapid transfer and shortening this time cost for passengers are important in high-speed rail transport organization.

\section{Coordination demands of high-speed and conventional rails}

According to the features of rail networks, at the beginning of construction and operation of high-speed lines, the mode of 'division-cooperation' between highspeed and conventional lines is key to better transport capacity within the rail network.

\subsection{Organization of cross-line trains}

The means of passenger transfer and the cross-line transport will have a certain impact on the coordination between high-speed and conventional lines. In order to coordinate the operation between them, one should consider two main aspects:

(1) In transfer stations, high-speed and conventional trains should have a good connection in their arrival and departure time, which creates a high demand for punctuality. Since trains in conventional lines have many different levels, resulting in large speed deviation and complex transport organization, punctuality with conventional line dispatch is a challenge.

(2) When using cross-line transport, the needs of high-speed rail networks for high speed, high density, and high security demand a much more rigid timetable. Cross-line trains should avoid the comprehensive maintenance window time for high-speed lines, which limits, to some extent, the feasible range of conventional lines. Meanwhile, the optimal arrival and departure times of passenger trains makes it more difficult to operate crossline trains in conventional lines. If trains are delayed in conventional lines, it will be very difficult to cross into high-speed lines on time. If this cannot be handled properly, the delay on conventional lines will spread into high-speed lines, causing disorder in the operation of high-speed rail networks. Therefore, the punctuality of cross-line trains in conventional lines should be ensured, and it is necessary to prepare some reserved train paths during the process of railway timetable drawing.

\subsection{Coordination in the connection stations of high- speed and conventional lines}

According to experiences in the operation of highspeed lines, as long as high-speed lines are connected with other lines, the transport organization at the connection point should be addressed. For example, in France, because high-speed trains can be operated off high-speed lines, the main point of its transport organization lies in the coordination at the connection point of the extended and high-speed lines, but not in the highspeed lines themselves. In Germany, as high-speed hub stations have many leading-in directions, utilization of arrival and departure tracks and connection of trains turn out to be the focus of its transport organization. Therefore, in joining high-speed and conventional rail networks, the connection stations are one of the key points for transport coordination. Transport organization and coordination in connection stations is the major work of dispatch coordination between high-speed and conventional lines, including the following three aspects:

(1) Technique coordination. 
The interconnection between high-speed and conven-

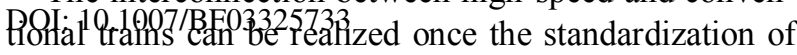
technical operation is fixed, which will provide good conditions for cross-line trains. The purpose of coordination is to ensure the optimal operation and best passing mode of passenger trains.

(2) Organization coordination.

High-speed and conventional lines should follow their own functions and ensure the smooth operation in daily transport production. Thus, one must establish the division methods of controlling authority for dealing with equipments of high-speed and conventional stations. This kind of problem exists mainly in hub stations of passenger transport. Based on previous studies, highspeed and conventional lines should both adopt two dispatch systems, independent, but interrelated. Since highspeed lines have been integrated into stations of conventional lines, there have been problems of jurisdiction division for the two systems. According to the operation of high-speed trains, two premises should be guaranteed on the study of jurisdiction division. First, there must be certain connecting high-speed running paths on the high-speed lines that should not be disturbed by any other operation or shunting work. Second, the dispatch system of high-speed lines, especially the command authority for train-running, should be consecutive. Trains running on high-speed lines should always be under the control of the high-speed dispatch system

(3) Information coordination.

In order to realize a favorable interconnection between high-speed and conventional rail systems, interoperability of information must be ensured so that resource sharing can be available and operation efficiency can be improved. Information coordination requires the consistency of information between the two rail systems. In the subsystems of connection stations, standards for input and output of information should be uniform. The method of providing information should be uniform, and one subsystem should provide information rapidly and timely, so that other corresponding subsystems can respond quickly.

\section{Optimization model of dispatch coordi- nation}

Compared with conventional lines, high-speed lines are only open for passenger trains. There are not only high-speed trains of class $\mathrm{A}$ at speeds of more than $300 \mathrm{~km} / \mathrm{h}$ and class $B_{1}$ at speeds of $250 \mathrm{~km} / \mathrm{h}$, but also cross-line trains of class $\mathrm{B}_{2}$. Affected by trains in conventional lines, cross-line trains may deviate from the planned operation line while entering high-speed stations, resulting in interference with trains in high-speed lines. High-speed train operation adjustment means to create a comprehensive organization of self-line and cross-line trains, making the latter operate safely and efficiently under the premise of guaranteeing the normal operation of the former.

As up-line and down-line trains do not disturb each other on high-speed lines, we take down-line trains for illustration in this paper.

Variable description: $T_{\mathrm{s}}$ is the starting time of adjustment phase; $T_{\mathrm{e}}$ is the end time of adjustment phase; $T_{D}^{H}$ is the ensemble of trains of class A during the adjustment phase, and $N_{D}^{H}=\left|T_{D}^{H}\right|, i=1,2, \cdots, N_{D}^{H} ; T_{D}^{M}$ is the ensemble of trains of class $B_{1}$ during the adjustment phase, and $N_{D}^{M}=\left|T_{D}^{M}\right|, i=1,2, \cdots, N_{D}^{M} ; T_{D}^{O}$ is the ensemble of trains of class $B_{2}$ during adjustment phase, and $N_{D}^{O}=\left|T_{D}^{O}\right|, i=1,2, \cdots, N_{D}^{O} ; T_{D}$ is the ensemble of trains during the adjustment phase: $T_{D}=T_{D}^{H} \cup T_{D}^{M} \cup T_{D}^{O}$; $N_{D}=\left|T_{D}\right|, i=1,2, \cdots, N_{D} ; \omega_{i}$ is the priority value of train $i$ during the adjustment phase, $\forall i \in N_{D}$, satisfying $\sum_{i \in T_{D}} w_{i}=1 ; \lambda(i)$ is the type of train $i$, where $\lambda(i)=1$ denotes the train of class $\mathrm{A}, \lambda(i)=2$ the train of class $B_{1}$, and $\lambda(i)=3$ the train of class $B_{2}, \forall i \in N ; S_{D}$ is the ensemble of stations, where stations are numbered one by one along the down-line direction, and $K_{D}=\left|S_{D}\right|$, $k=1,2, \cdots, K_{D} ; B_{D}$ is the ensemble of intervals, where intervals are numbered one by one along the down-line direction, and $K_{D}-1=\left|B_{D}\right|, \bar{k}=1,2, \cdots, K_{D}-1 ; G_{D}^{i}$ is the set of train paths according to the train operation plan: $\quad G_{D}^{i}=\left\{K_{1}^{i}, K_{2}^{i}, \cdots, K_{K(i)}^{i}\right\}, \quad \forall i \in T_{D}, \quad G_{D}^{i} \subseteq S_{D}$, where $K_{t}^{i}$ and $K_{K(i)}^{i}$ are, respectively, station $t$ and the last station passed by train $i$ according to the train operation plan; $J_{D}^{i}$ is the operation routine of train $i$ during the adjustment phase: $J_{D}^{i}=\left\{k_{1}^{i}, k_{2}^{i}, \cdots, k_{K(i)}^{i}\right\}, \forall i \in T_{D}, J_{D}^{i} \subseteq S_{D}$, where $k_{t}^{i}$ and $k_{K(i)}^{i}$ are, respectively, station $t$ and the last station passed by train $i$ during the adjustment phase; $t_{D}^{\lambda(i), k k}$ is the minimum net-operating time of trains of class $\lambda(i)$ during interval $\bar{k}, \forall i \in T_{D}, \forall \bar{k} \in B_{D} ; s_{D}^{i, k}$ is the minimum stop time of train $i$ in station $k$, and if train $i$ starts or arrives in station $k$, its minimum stop time equals zero, $\forall i \in T_{D}, \forall k \in G_{D}^{i} ; y_{D}^{i, k}$ is the scheduled arrival time of train $i$ at station $k$, and if train $i$ starts from station $k$, its scheduled arrival time equals scheduled departure time, $\forall i \in T_{D}, \forall k \in G_{D}^{i} ; y_{D}^{i, k}$ is the 
scheduled departure time of train $i$ at station $k$, and if ProIf 1.0 atrives $\$ \mathrm{BFP} 3325.733$, its scheduled departure time equals scheduled arrival time, $\forall i \in T_{D}, \forall k \in G_{D}^{i} ; \phi_{D}^{i, k}$ is a $0-1$ variable, if down-line train $i$ passes by downline station $k$, it is valued 0 , and otherwise $1, \forall i \in T_{D}$, $\forall k \in J_{D}^{i} ; \alpha_{D}^{\lambda(i), k}$ is the additional starting time of trains of class $\lambda(i)$ at station $k, \forall i \in T_{D}, \forall k \in S_{D} ; \beta_{D}^{\lambda(i), k+1}$ is the additional stopping time of trains of class $\lambda(i)$ at station $k+1, \forall i \in T_{D}, \forall k \in S_{D} ; c_{D}^{i, k}$ is a $0-1$ variable, if train has passenger service at station $k$, it is valued 1 , and otherwise $0, \forall i \in T_{D}, \forall k \in J_{D}^{i} ; X_{D}^{i, k}$ is the actual arrival time of train $i$ at station $k, \forall i \in T_{D}, \forall k \in J_{D}^{i}$; $Y_{D}^{i, k}$ is the actual departure time of train $i$ at station $k$, $\forall i \in T_{D}, \forall k \in J_{D}^{i} ; x_{D}^{i, k}$ is the planned arrival time of train $i$ at station $k, \forall i \in T_{D}, \forall k \in G_{D}^{i} ; y_{D}^{i, k}$ is the planned departure time of train $i$ at station $k, \forall i \in T_{D}$, $\forall k \in J_{D}^{i} . H_{D}^{i, K_{K(i)}^{i}}$ is the allowed earliest arrival time of the cross-line trains at the terminal station $K_{K(i)}^{i}$. According to the train levels, punctuality of arrival and departure, and requirements for travel time, we choose the minimum weighted deviation of trains' arrival and departure time as the optimization objective, by introducing $\Delta_{D}^{i, k}$ as the deviation of the actual arrival time and scheduled arrival time of train $i$ at station $k$ : $\Delta_{D}^{i, k}=\left|X_{D}^{i, k}-x_{D}^{i, k}\right| \nabla_{D}^{i, k}$ as the deviation of actual departure time and scheduled departure time for train $i$ at station $k: \nabla_{D}^{i, k}=\left|Y_{D}^{i, k}-y_{D}^{i, k}\right|$, as follows:

$$
\min z=\sum_{i \in N_{D}} \omega_{i} \sum_{k \in J_{D}^{i}}\left(\Delta_{D}^{i, k}+\nabla_{D}^{i, k}\right),
$$

s.t.

$$
\begin{gathered}
X_{D}^{i, k+1} \geq Y_{D}^{i, k}+t_{D}^{\lambda(i), \bar{k}}+ \\
\phi_{D}^{i, k} \alpha_{D}^{\lambda(i), k}+\phi_{D}^{i, k+1} \beta_{D}^{\lambda(i), k+1}, \\
c_{D}^{i, k k}=0 \text { and } c_{D}^{i, k+1}=0, \forall i \in T_{D}, \forall \bar{k} \in V_{D}^{i} ; \\
X_{D}^{i, k+1} \geq X_{D}^{i, k}+\phi_{D}^{i, k} \alpha_{D}^{\lambda(i), k}+\left(t_{D}^{\lambda(i), \bar{k}}+\beta_{D}^{\lambda(i), k+1}\right), \\
c_{D}^{i, k k}=0 \text { and } c_{D}^{i, k+1}=1, \forall i \in T_{D}, \forall \bar{k} \in V_{D}^{i} ; \\
X_{D}^{i, k+1} \geq Y_{D}^{i, k}+\left(t_{D}^{\lambda(i), \bar{k}}+\alpha_{D}^{\lambda(i), k}\right)+\phi_{D}^{i, k+1} \beta_{D}^{\lambda(i), k+1}, \\
c_{D}^{i, k}=1 \text { and } c_{D}^{i, k+1}=0, \forall i \in T_{D}, \forall \bar{k} \in V_{D}^{i} ; \\
X_{D}^{i, k+1} \geq Y_{D}^{i, k}+\left(t_{D}^{\lambda(i), \bar{k}}+\alpha_{D}^{\lambda(i), k}+\beta_{D}^{\lambda(i), k+1}\right), \\
c_{D}^{i, k}=1 \text { and } c_{D}^{i, k+1}=1, \forall i \in T_{D}, \forall \bar{k} \in V_{D}^{i} ;
\end{gathered}
$$

$$
\begin{gathered}
H_{D}^{i, k_{k(i)}^{i}} \leq X_{D}^{i, k_{k(i)}^{i}} \leq Q_{D}^{i, k_{k(i)}^{i}}, \\
\forall i \in T_{D}^{O} \text { and } K_{k(i)}^{i} \in J_{D}^{i} .
\end{gathered}
$$

Among the above expressions, (1) is the objective function, which will minimize the deviation of trains' arrival and departure time; (2) represents constraints of running time in sections; (3) represents constraints of stop time at stations; (4) represents constraints of headway; (5) represents constraints of the earliest feasible departure time; and, (6) represents constraints of crossline time of cross-line trains.

Among the exact algorithms at present, branch-andbound and cutting-plane are considered two effective algorithms for resolving the problem. Because it is difficult to find the cutting plane, it is proposed this model be resolved by the branch-and-bound algorithm.

\section{Establishment of organization}

In order to achieve the dispatch coordination between high-speed and conventional lines, functional coordination and information sharing should be taken into consideration. As conventional lines in China are freightoriented, the existing dispatch system is made up of four main types of work: train operation, locomotives, freight, and plan. However, the dispatch on the high-speed lines mainly targets passenger trains. Therefore, it focuses on passenger-oriented service. Based on this, conventional rails should independently control the freight dispatch systems (freight-DS), locomotives-DS, and planning-DS, while high-speed rails should not only establish its own dispatch system to satisfy its business requirements, but also consider as well the sharing of certain dispatch systems with conventional rails, such as train operation and management-DS, EMU-DS, and passenger service-DS. The power supply-DS and comprehensive maintenance system, under the commanded of high-speed dispatch centre, correspond respectively to the power supply dispatch room and engineering affairs dispatch room of conventional lines. Therefore, the corresponding dispatch platforms of high-speed and conventional lines include train operation and management-DS, EMU-DS, passenger service-DS, power supply-DS, and comprehensive maintenance system. In the high-speed rail dispatch system, the dispatch centre controls directly all the dispatch platforms and just provides information for these platforms for the conventional rail dispatch centre. The organization establishment of high-speed and conventional rail dispatch systems is shown in Fig. 1.

In Fig. 1, the solid arrow indicates direct control, and the dotted arrow indicates information exchange. Similarly, in the conventional rail dispatch system, in addition to planning-DS, locomotive-DS, and freight-DS, the dispatch centre in conventional stations should also es- 
tablish the train operation and management-DS, EMUBSI: passenger Service-D3S, power supply-DS, and comprehensive maintenance system. These five systems are also controlled by the dispatch centre in railway stations, and provide information for the high-speed rail dispatch centre.

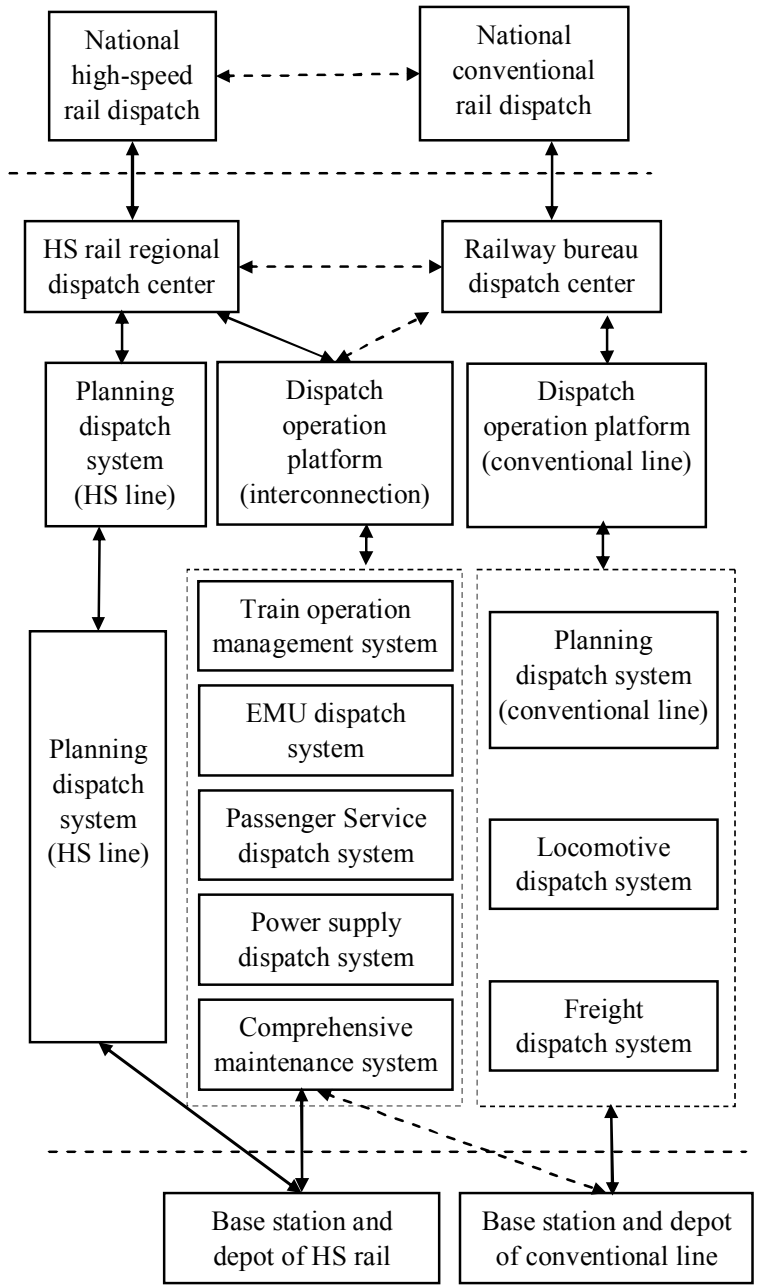

Fig. 1 Organization establishment of dispatch system between high-speed and conventional lines

\section{Coordination}

The key point of dispatch coordination lies in the transport organization of cross-line trains and the dispatch of work in connection stations. Dispatch coordination between high-speed and conventional lines is shown in Fig. 2. In Fig. 2, arrows that start from the related department of the dispatch centre to the corresponding department or task involved in dispatch indicate that this department is in charge of a certain dispatch task; bidirectional arrows represent information exchange during the execution of different tasks. The dispatch of train operation and management, EMU, and passenger service are key sections of cross-line train op- eration and work organization in connection stations. Through coordination between dispatch systems of high-speed and conventional lines, the information exchange of cross-line trains and the proper interconnection of command on all lines can be achieved. A solid arrow indicates the direct influence or control while the dotted arrow indicates information exchange.

In Fig. 2, letters from $A$ to $W$ are defined as follows:

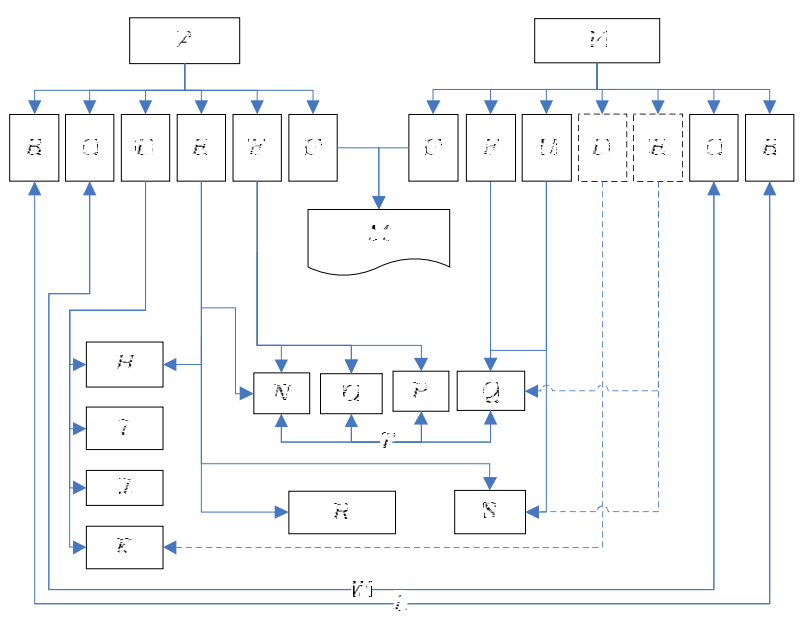

Fig. 2 Dispatch coordination between high-speed and conventional lines

$A$ : Regional dispatch center of high-speed rail.

$B$ : Comprehensive maintenance dispatch.

$C$ : Power supply control.

$D$ : EMU dispatch.

$E$ : Passenger service dispatch.

$F$ : Train operation management dispatch.

$G$ : Train operation plan management.

$H$ : Cross-line EMU routing plan adjustment.

I: Vehicle-allocation plan adjustment, crew plan adjustment, and maintenance plan adjustment.

$J$ : Reserve EMUs in the operation depot and EMU maintenance base.

$K$ : Cross-line EMU monitoring.

$L$ : Intercommunication of signal, line-state, and safety-control information.

$M$ :Train-operation plan, EMU routing plan, vehicleallocation plan, crew plan, maintenance plan, comprehensive maintenance plan, power-supply plan, and station-operation plan.

$N$ : Train-operation adjustment in the high-speed rail zone.

$O$ : Technical operation in high-speed rail stations.

$P$ : Technical operation in conventional station (highspeed yard).

$Q$ : Train-operation adjustment in the conventional railway zone. 
$R$ : Passenger organization in the high-speed train staDOI: 101007 ThEP 325733 tion, ticket business, and indication system.

$S$ : Cross-line passenger organization on the conventional railway line, cross-line ticket business, and cross-line indication system.

$T$ : Cross-line train-operation information.

$U$ : Passenger transport dispatch.

$V$ : Railway bureau dispatch center.

$W$ : Intercommunication of power-supply information.

Jurisdictional division of these two dispatch systems must obey the 'single command' principle. One yard, track, access, or turnout group can only be controlled by one system, and monitored by another system. The dispatch staff in base stations can only be commanded by one related dispatch platform of one system.

In connection stations, the work related to the trainroute of high-speed trains are operated by the dispatch system of high-speed rails, while others are operated by that of conventional rails. As to cross-line trains, the trainroute of getting on and off high-speed lines is controlled by those in charge of receiving trains; that is, the trainroute of getting on high-speed lines is controlled by the high-speed dispatch system, while that of getting off is controlled by the conventional lines dispatch system.

In unexpected situations, train operation needs to be adjusted and the process of dispatch coordination of high-speed and conventional lines is shown in Fig. 3.

The passenger service dispatch of high-speed lines and the passenger transport dispatch of conventional lines are managed and fed back according to passenger flows, ticket affaires, and other information of cross-line trains (as shown by "1" in Fig. 3). Train operation, technical operation in stations in high-speed rail sections, and the work related to the high-speed station yard in conventional stations are organized and adjusted in the same time by the dispatch of passenger service, train

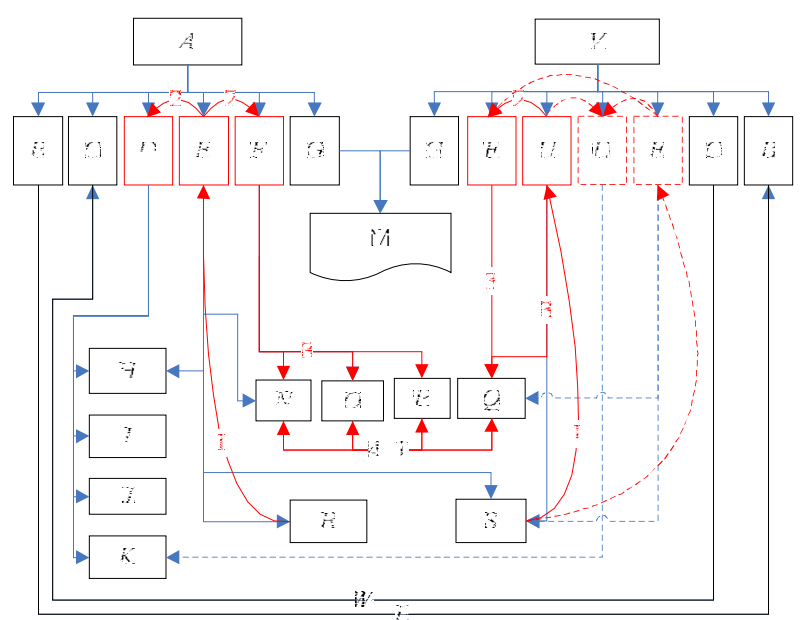

Fig. 3 Coordination process in unexpected situations operation and management, and EMU of high-speed lines. Trains on conventional lines are organized and adjusted in the same time by the dispatch of passenger transport and that of train operation and management (as shown by "2" and "3" in Fig. 3). Via sharing and transferring the operation information of cross-line trains, the dispatch coordination between high-speed and conventional rails can be realized (as shown by "4" in Fig. 3).

\section{Conclusions}

Operation of cross-line trains and work coordination in connection stations are key tasks of dispatch coordination between high-speed and conventional rails. By analyzing the necessity of dispatch coordination between high-speed and conventional lines, this paper builds an adjustment model for high-speed train operation with the operation of cross-line trains taken into consideration. The dispatch organization of high-speed and conventional rails is set up according to the operation of cross-line trains and the work coordination in connection stations. The dispatch coordination between high-speed and conventional lines is described, and the process of coordination adjustment in unexpected situations is analyzed.

This research provides a certain theoretical basis for building a high-speed rail dispatching system. The detailed settings and function segmentations of dispatch organization, and system design and development remains as the main work of optimization of high-speed rail dispatch.

\section{Acknowledgements}

This research is one of the key parts of an NNFF (National Natural Science Foundation) project under grant 60776827: 'Train network operation program with optimization theory and method research', and meanwhile is the key research in 'Study of optimization method and adjustment theory of high-speed train operation' supported by the Doctoral Program Foundation of Ministry of Education under grant 20090184110011.

\section{References}

[1] B. Szpigel, Optimal train scheduling on a single line railway, Operations Research, 1973, 27(5): 344-351.

[2] D. Jovanovicv, Improving railroad on-time performance models, Algorithms and Applications [Dissertation]. Philadelphia, PA : University of Pennsylvania, 1989.

[3] S. Araya, K. Abe, An optimal rescheduling for online train traffic control in disturbed situation, In: J.L. Melsa, ed, In: Proc. of 22nd IEEE Conf .on Decision and Control, San Antonio, 1983, 489-494. 
[4] J.M. Cao, Optimization model for adjusting diagram on

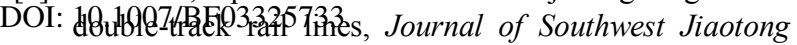
University, 1995, 30(5): 520-526 (in Chinese).

[5] L.W. Xiao, R. H. Xie, Automatic adjustment of train operation diagram in single-line block, Journal of Traffic and Transportation Engineering, 2004, 4(3): 25-29 (in Chinese).

[6] Q. Luo, F.C. Jin, S. J. Hu, Decomposition coordination computing model for train operation adjustment, Journal of Beijing Jiaotong University, 2004, 28(6): 87-89, 94 (in Chinese).

[7] Y.R. Chen, Q.Y. Peng, Y.S. Jiang, Research on a model for adjusting train diagram on double-track railway with satisfactory optimization, Journal of China Railway Society, 2003, 25(3): 8-12 (in Chinese).

[8] L. Lei, X.C. Zhang, P. Zhao, et al., Study on the strategy of train operation adjustment on high-speed railway, Journal of China railway Society, 2001, 23(8): 1-6 (in Chinese).

[9] X.S. Xie, A Study on Train Traffic Control of Passenger Traffic Special Line [Dissertation], Chengdu: Southwest Jiaotong University, 2009 (in Chinese).

[10] H.W. He. Ballastless Technology, Beijing: China Railway Publishing House, 2005 (in Chinese).

(Editor: Junsi LAN) 